\title{
Field sparsening for the construction of the correlation functions in lattice QCD
}

\author{
Yuan Li®, ${ }^{1}$ Shi-Cheng Xia, ${ }^{1}$ Xu Feng $\odot,{ }^{1,2,3,{ }^{*}}$ Lu-Chang Jin $\odot,{ }^{4,5, \dagger}$ and Chuan Liu ${ }^{1,2,3}$ \\ ${ }^{1}$ School of Physics and State Key Laboratory of Nuclear Physics and Technology, \\ Peking University, Beijing 100871, China \\ ${ }^{2}$ Collaborative Innovation Center of Quantum Matter, Beijing 100871, China \\ ${ }^{3}$ Center for High Energy Physics, Peking University, Beijing 100871, China \\ ${ }^{4}$ Department of Physics, University of Connecticut, Storrs, Connecticut 06269, USA \\ ${ }^{5}$ RIKEN-BNL Research Center, Brookhaven National Laboratory, \\ Building 510, Upton, New York 11973, USA
}

(Received 9 September 2020; accepted 5 January 2021; published 22 January 2021)

\begin{abstract}
Two field-sparsening methods, namely the sparse-grid method and the random field selection method, are used in this paper for the construction of the 2-point and 3-point correlation functions in lattice QCD. We argue that, due to the high correlation among the lattice correlators at different field points associated with source, current, and sink locations, one can save a lot of computational time by performing the summation over a subset of the lattice sites. Furthermore, with this strategy, one only needs to store a small fraction of the full quark propagators. It is found that the number of field points can be reduced by a factor of $\sim 100$ for the point-source operator and a factor of $\sim 1000$ for the Gaussian-smeared operator, while the uncertainties of the correlators only increase by $\sim 15 \%$. Therefore, with a modest cost of the computational resources, one can approach the precision of the all-to-all correlators using the field-sparsening methods.
\end{abstract}

DOI: 10.1103/PhysRevD.103.014514

\section{INTRODUCTION}

Lattice QCD provides a nonperturbative approach to the numerical solution of Quantum Chromodynamics (QCD), which is believed to be the basic theory of strong interactions among quarks and gluons. With the development of the cutting-edge supercomputers, the new algorithms and the advanced methodologies, lattice QCD is now playing an increasingly important role in the understanding of low-energy QCD.

A typical way to gain a better efficiency in a numerical lattice QCD calculation is to reduce the redundant costs. Here are some examples.

(i) To guarantee an accurate generation of a quark propagator, the residual criterion in the conjugate gradient (CG) inversion for the quark propagator is usually set to $10^{-8}$ or even smaller. When realizing that most of the CG iterations can be saved, the all mode averaging (AMA) technique [1] is proposed, where the residual criterion is raised to a level of

\footnotetext{
*xu.feng@pku.edu.cn

†ljin.luchang@gmail.com
}

Published by the American Physical Society under the terms of the Creative Commons Attribution 4.0 International license. Further distribution of this work must maintain attribution to the author(s) and the published article's title, journal citation, and DOI. Funded by SCOAP. $\sim 10^{-4}$. In this way the computational time is significantly reduced while the physical quantities can still be obtained with no bias by performing a correction, which compensates the systematic effects from the approximated propagators by adding the difference between some samples of the precise correlators and the approximated ones.

(ii) In the calculation of hadronic light-by-light contributions to the muon anomalous magnetic moment, a four-point hadronic function with vector-currents is required. To construct such a four-current correlator, one expects a spacetime summation over the locations of at least three of the four currents which is very challenging numerically. Realizing that in the connected diagram, when the locations of two vector currents are separated with a spacetime distance $r$, the hadronic function falls exponentially with the increase of $r$, an importance sampling is introduced to evaluate the stochastic sum over $r$ efficiently [2]. Therefore, in the important regions where $r \lesssim 1 \mathrm{fm}$, the summation is run in a complete way while in the other regions $r>1 \mathrm{fm}$, the contributions are calculated with a probability of $p(r) \propto 1 / r^{3.5}$. In this way, much less computational resources are spent in the very long distance region, where the lattice correlation functions mainly contribute noise rather than the signal.

(iii) In many cases, it is appealing to utilize the translational invariance and construct the correlation 
function using the all-to-all propagators [3-6]. As a result, the information over the whole spacetime volume is summed and one expects to gain a good precision for the correlation function. On the other hand, generating all-to-all propagators is quite time consuming. Since the correlation functions from the same configuration is highly correlated, one can achieve nearly the same precision by averaging over part of the source and sink locations. Such kind of techniques, called field-sparsening methods here, are the main focus of this paper.

The field-sparsening techniques have been studied by Detmold and Murphy in Ref. [7], where a sparse-grid technique is introduced. An earlier application of the sparse grid can be traced back to a study from $\chi \mathrm{QCD}$ collaboration in 2010 [8]. In this work, in addition to the sparse-grid approach, we developed another field-sparsening approach, which we will refer to as the random field selection method. We will study and compare these two methods in some detail. The paper is organized as follows. We start with Sec. II by introducing the two field-sparsening techniques mentioned above. In Sec. III we discuss the results of the 2-point functions for pion and proton with both pointsource and Gaussian-smeared-source operators. The advantages and disadvantages for each field-sparsening method are also discussed. In Sec. IV we employ a simple model to analyze the sources of the uncertainties for the random field selection method. In Sec. V we extend the study to the 3-point function, where the proton axial charge $g_{A}$ is used as an example to demonstrate the efficiency of the fieldsparsening methods.

\section{FIELD-SPARSENING METHODS}

In the lattice QCD calculation of a generic n-point function,

$$
\sum_{\vec{x}_{1}, \vec{x}_{2}, \ldots, \vec{x}_{n-1} \in \Lambda_{\text {full }}}\left\langle O_{1}\left(x_{1}\right) O_{2}\left(x_{2}\right) \cdots O_{n}\left(x_{n}\right)\right\rangle,
$$

with $O_{i}\left(x_{i}\right)$ being the interpolating operators at temporalspatial point $x_{i}=\left(x_{i}^{0}, \vec{x}_{i}\right)$ and $\Lambda_{\text {full }}$ the full set of spatial lattice points, one needs to perform the volume summation $(n-1)$ times. This results in a computational cost of $\left(L^{3}\right)^{n-1}$ in the quark contraction, with $L$ being the spatial lattice size. If one wants to gain a better precision by making another spatial-volume average over the locations of $x_{n}$, then the cost becomes $\left(L^{3}\right)^{n}$. The typical size of $L^{3}$ is about $10^{4}-10^{6}$ for practical lattice QCD simulations. Given a relatively large lattice, the complexity of $O\left(L^{6}\right)$ usually exceeds the capability of the current lattice QCD calculations. In many cases, the techniques of all-to-all propagators [6] or sequential-source propagators [9] are used to reduce the computational costs. On the other hand, there are some limitations for the usage of these propagators.
For example, the all-to-all propagators work more efficiently in the mesonic sector than in the baryonic sector [10]. For the sequential-source propagators, although it allows to reduce a complexity of $O\left(L^{6}\right)$ to a level of $O\left(L^{3}\right)$, the computational cost can increase dramatically if one wants to build the sequential-source propagators with multiple time slices, momentum insertions and gamma matrix structures.

Given a gauge configuration from Monte Carlo simulation, the correlation functions at different source and sink locations are usually highly correlated statistically. Therefore one can save computational time by only summing over a small subset of all the possible source or sink locations. In fact, as will be shown below, utilizing less source locations one can efficiently reduce the costs to generate the quark propagators. For each propagator, one can use less sink locations and reduce the computational cost for the Wick contractions in the construction of the correlation functions. Since the numbers of both source and sink locations are reduced, it also saves the disk space to store these quark propagators and also reduces the pressure for the input and output (I/O) of the large-size data on the supercomputers. It is the task of this paper to show how much fewer location points one can use to maintain a comparable precision of the correlation functions that use the full location points.

For simplicity, we start with the 2-point correlation function as an example to introduce the field-sparsening techniques. The standard 2-point correlation function with zero spatial momentum insertion is written as

$$
C_{\text {full }}(t)=\sum_{\vec{x} \in \Lambda_{\text {full }}}\left\langle O\left(\vec{x}, t_{0}+t\right) O^{\dagger}\left(\vec{x}_{0}, t_{0}\right)\right\rangle,
$$

where the subscript "full" indicates that the summation of $\vec{x}$ runs over all the sink location points. By using field sparsening, one can replace the summation $\sum_{\vec{x} \in \Lambda_{\text {full }}}$ by $\frac{L^{3}}{N_{\Lambda}} \sum_{\vec{x} \in \Lambda}$, where $\Lambda$ is a subset of $\Lambda_{\text {full }}$, which contains only $N_{\Lambda}$ location points. Due to the high correlation in the lattice data, we expect that the replacement does not increase the noise much but reduces the propagator storage and contraction time for modest size $N_{\Lambda}$. In Eq. (2) only one source location $\left(\vec{x}_{0}, t_{0}\right)$ is used. In principle one can use multiple source locations and write the correlation functions as

$$
\begin{aligned}
C_{\text {sparse }}(t)= & \frac{L^{3}}{N_{\Lambda} N_{\Lambda_{0}} N_{\Lambda_{t}}} \sum_{\vec{x} \in \Lambda} \sum_{\vec{x}_{0} \in \Lambda_{0}} \sum_{t_{0} \in \Lambda_{t}} \\
& \times\left\langle O\left(\vec{x}, t_{0}+t\right) O^{\dagger}\left(\vec{x}_{0}, t_{0}\right)\right\rangle,
\end{aligned}
$$

where the source spatial location takes the value from the set $\Lambda_{0}$ and source time slice takes the value from $\Lambda_{t}$. The size of the set $\Lambda_{0}$ and $\Lambda_{t}$ is given by $N_{\Lambda_{0}}$ and $N_{\Lambda_{t}}$, respectively. In this work we will compare two different sets for $\Lambda\left(\Lambda_{0}\right)$, namely the sparse-grid method and the random field selection method, and determine the optimal values for $N_{\Lambda}$ and $N_{\Lambda_{0}}$ in each case. 


\section{A. Sparse-grid method}

Following the sparse-grid method introduced in Ref. [7], the set $\Lambda$ is chosen as,

type I: $\left\{\left(n_{1}, n_{2}, n_{3}\right) \mid 0 \leq n_{i}<L ; n_{i}=0(\bmod k)\right\}$, where $k$ is an integer factor of $L$. By using this setup, a $L^{3}$ point spatial lattice is reduced to a $(L / k)^{3}$-point one. For all the time slices $t_{0}$ and $t_{0}+t$ used in Eq. (2), the same sparse grid set is implemented. One can also extend the type of the sparse grid to

$$
\begin{array}{r}
\text { type II: }\left\{\left(n_{1}, n_{2}, n_{3}\right) \mid 0 \leq n_{i}<L ; n_{i}=0(\bmod k) ; n_{1}+n_{2}+n_{3}=0(\bmod 2 k)\right\}, \\
\text { type III: }\left\{\left(n_{1}, n_{2}, n_{3}\right) \mid 0 \leq n_{i}<L ; n_{i}=0(\bmod k) ; n_{i}+n_{j}=0(\bmod 2 k), i \neq j\right\} .
\end{array}
$$

With the above definitions, we have $N_{\Lambda}=\frac{L^{3}}{k^{3}}, \frac{L^{3}}{2 k^{3}}, \frac{L^{3}}{4 k^{3}}$ for type I, II and III, respectively. In our numerical study, we use a lattice gauge ensemble with $L=24$ and pick up 15 values for $N_{\Lambda}$. For convenience, these 15 cases are labeled by an integer denoted as $N_{\text {th }}$, running from 0 to 14 , and the corresponding values for $N_{\Lambda}$ are given by the following list:

$$
N_{\Lambda}\left(N_{\text {th }}\right)=\left\{24^{3}, 12^{3}, \frac{12^{3}}{2}, \frac{12^{3}}{4}, 6^{3}, \frac{6^{3}}{2}, 4^{3}, \frac{6^{3}}{4}, \frac{4^{3}}{2}, 3^{3}, \frac{4^{3}}{4}, 2^{3}, 4,2,1\right\} .
$$

It means that we have $N_{\Lambda}=24^{3}$ for $N_{\text {th }}=0$ and $N_{\Lambda}=1$ for $N_{\text {th }}=14$.

Note that the sets of type I, II, III always include the location point of $\left(n_{1}, n_{2}, n_{3}\right)=(0,0,0)$. We therefore can consider it as a reference point. To reduce the correlation in the lattice calculation, for each configuration, one can shift the reference point randomly with $L^{3} / N_{\Lambda}$ choices.

\section{B. Random field selection}

Another choice of $\Lambda$ is called random field selection, with $\Lambda$ chosen as

$\Lambda=\left\{\left(n_{1}, n_{2}, n_{3}\right) \mid 0 \leq n_{i}<L ; n_{i}\right.$ are random numbers $\}$.

The random numbers for $n_{i}$ vary when the time slice $t$ and configuration trajectory change. In principle, we can use any value for $N_{\Lambda}$. To favor a comparison with the sparsegrid method, we use the same choices of $N_{\Lambda}$ as that in Eq. (6).

\section{2-POINT FUNCTION}

In this section we will present the results for 2-point correlation functions. The calculation is performed using a gauge ensemble with $2+1+1$ clover-improved Wilson twisted mass quarks, generated by the ETM Collaboration [11]. The lattice volume is $24^{3} \times 48$ with a pion mass $m_{\pi} \approx$ $350 \mathrm{MeV}$ and a lattice spacing $a \approx 0.093 \mathrm{fm}$. In total 91 configurations are utilized in this analysis. For simplicity, all the results presented in this paper are given in lattice units unless specified.

We use Gaussian-smeared-source propagators to construct the smeared-source smeared-sink 2-point functions

$$
C_{\alpha}(t)=\frac{L^{3}}{N_{\Lambda} N_{\Lambda_{0}} N_{\Lambda_{t}}} \sum_{\vec{x} \in \Lambda} \sum_{\vec{x}_{0} \in \Lambda_{0}} \sum_{t_{0} \in \Lambda_{t}}\left\langle O_{\alpha}\left(\vec{x}, t_{0}+t\right) O_{\alpha}^{\dagger}\left(\vec{x}_{0}, t_{0}\right)\right\rangle,
$$

with $\alpha=\pi$ for pion and $\alpha=p$ for proton. We use 24 time slices for $t_{0}$. For each time slice, we use 8 random source locations for $\vec{x}_{0}$. Thus we have $N_{\Lambda_{t}}=24$ and $N_{\Lambda_{0}}=8$. For the sink location $\vec{x}$, it can be summed over the full set $\Lambda_{\text {full }}$ or field-sparsening set $\Lambda$. Figure 1 shows the effective masses for the pion (left panel) and the proton (right panel) with the point set $\Lambda_{\text {full }}$ and $\Lambda$ at $N_{\Lambda}=2^{3}$. The effective masses $m_{\alpha}$ at the time slice $t$ are obtained using $C_{\alpha}(t)$ and $C_{\alpha}(t+1)$ as inputs. For the two field-sparsening methods, the data points are slightly shifted horizontally to favor a comparison with that from the full set.

From Fig. 1 a clear enhancement of the excited-state contamination is found in the sparse-grid method at $N_{\Lambda}=2^{3}$. This is due to the mixing of the hadron states with high momenta. Let us take the sparse-grid set type I as an example. The summation over $\Lambda$ can be written as

$$
\frac{L^{3}}{N_{\Lambda}} \sum_{\vec{x} \in \Lambda}=\sum_{\vec{m} \in \Gamma} \sum_{\vec{x} \in \Lambda_{\text {full }}} e^{i \frac{2 \pi}{k} \vec{m} \cdot \vec{x}},
$$

with $\Gamma=\left\{\left(m_{1}, m_{2}, m_{3}\right) \mid m_{i}=0,1, \ldots, k-1\right\}$. Therefore the higher-momentum modes with $\vec{m} \neq \overrightarrow{0}$ mix with the zero-momentum mode. As a consequence, the excited-state contaminations increase as $N_{\Lambda}$ decreases. The situation becomes even more problematic when one targets on the calculation of the correlation functions with large momentum transfer. In this case it is possible that a state with an 

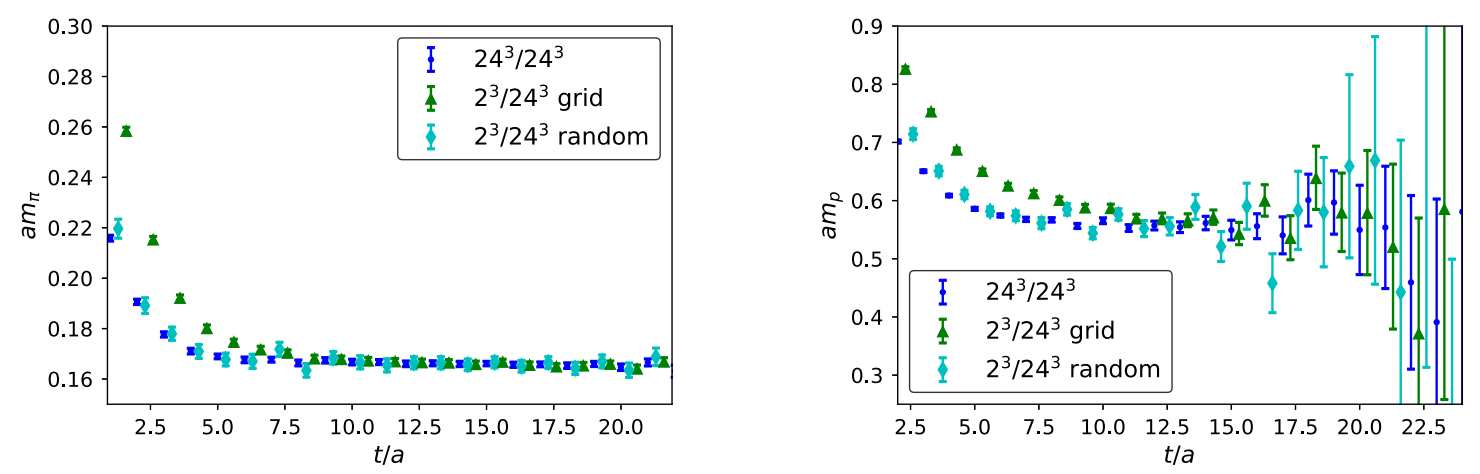

FIG. 1. Effective masses for the pion (left panel) and the proton (right panel) with full set $\Lambda_{\text {full }}$ (blue), sparse-grid (dark green) and random-field selection (cyan). For the two field-sparsening methods, we use $N_{\Lambda}=2^{3}$. The green and cyan data points are shifted horizontally for an easier comparison.

assigned momentum mixes with the states carrying smaller momenta and consequently the correlation functions are distorted by the low-lying states.

For the random-field selection method, there is no enhancement of the excited-state contribution. On the other hand, the statistical errors become larger due to the random noise arising from the field sparsening. Note that the correlation among $C_{\alpha}(t)$ at various $t$ is weakened by the random-field selection. As a result, the uncertainty for the effective mass can be further reduced if one performs a fit over a temporal window.

In Fig. 2, we show the effective mass from the correlated fit as a function of $N_{\text {th }}$, or equivalently the different choices of $N_{\Lambda}$. The fitting windows are determined using the data with $\Lambda_{\text {full }}$. To be specific, we use the fitting window $8 \leq$ $t \leq 22$ for the pion correlator and obtain $m_{\pi}=0.16632(46)$ from a correlated fit with $\chi^{2} /$ dof $=0.96$. We use the fitting window $10 \leq t \leq 22$ for the proton and obtain $m_{p}=$ $0.5548(44)$ with $\chi^{2} /$ dof $=0.35$. We denote these effective masses as $m_{\pi}^{\text {full }}$ and $m_{p}^{\text {full }}$. The reasonable values of $\chi^{2} /$ dof suggest that the excited-state contributions are well under control. We thus fix these fitting windows for the fieldselection cases. For the sparse-grid method, the additional

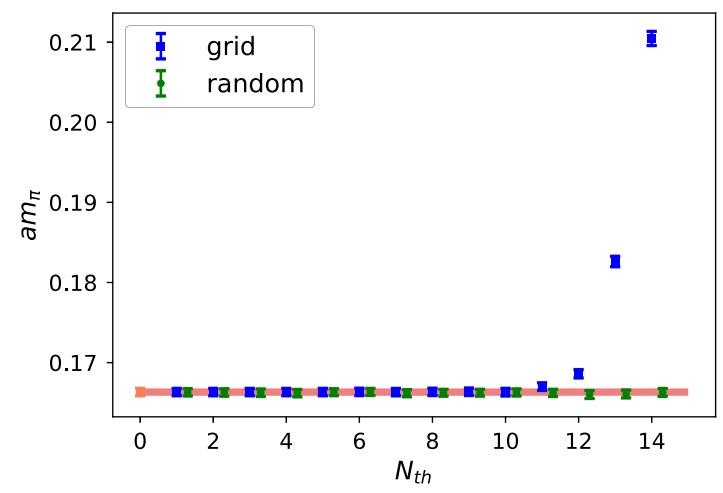

excited-state contributions from higher momenta start to make an obvious impact on the effective mass when $N_{\text {th }} \geq 11$ (or $N_{\Lambda} \leq 8$ ). For the random field selection, although the effective masses at each time slice carry larger errors, the uncertainties are very close to the ones from sparse-grid method after a correlated fit. We find that the effective masses at $N_{\text {th }}=10\left(N_{\Lambda}=16\right)$ are given by $m_{\pi}=0.16627(44)$ and $m_{p}=0.5553(51)$. By using the random field selection method, one can reduce in the summation the number of the sink points by a factor of 864 $\left(N_{\Lambda}=L^{3} \rightarrow 16\right)$, while the lattice results are still very precise. For the pion effective mass, the statistical uncertainty is consistent with that from $\Lambda_{\text {full }}$, and, for the proton, the uncertainty only increases by about $16 \%$.

The efficiency of the field sparsening likely depends on the type of interpolating operators used in the lattice calculation. As the Gaussian-smeared-source propagator is more correlated than the point-source propagator, we expect that the field sparsening works more efficiently for the former case. To confirm this conjecture we calculate the effective masses for the pion and proton using both Gaussian-smeared-source and point-source propagators. To save the cost, we keep $N_{\Lambda_{t}}$ at 24 while reducing $N_{\Lambda_{0}}$

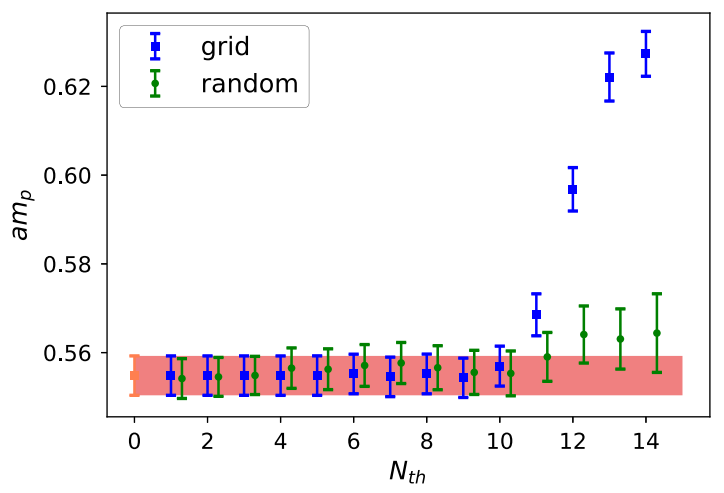

FIG. 2. Effective masses for the pion (left panel) and proton (right panel) from the correlated fit as a function of $N_{\text {th }}$. For each $N_{\text {th }}$, the number of field points is given in Eq. (6). The data points from sparse-grid method and random field selection are printed in blue and green color, respectively. 

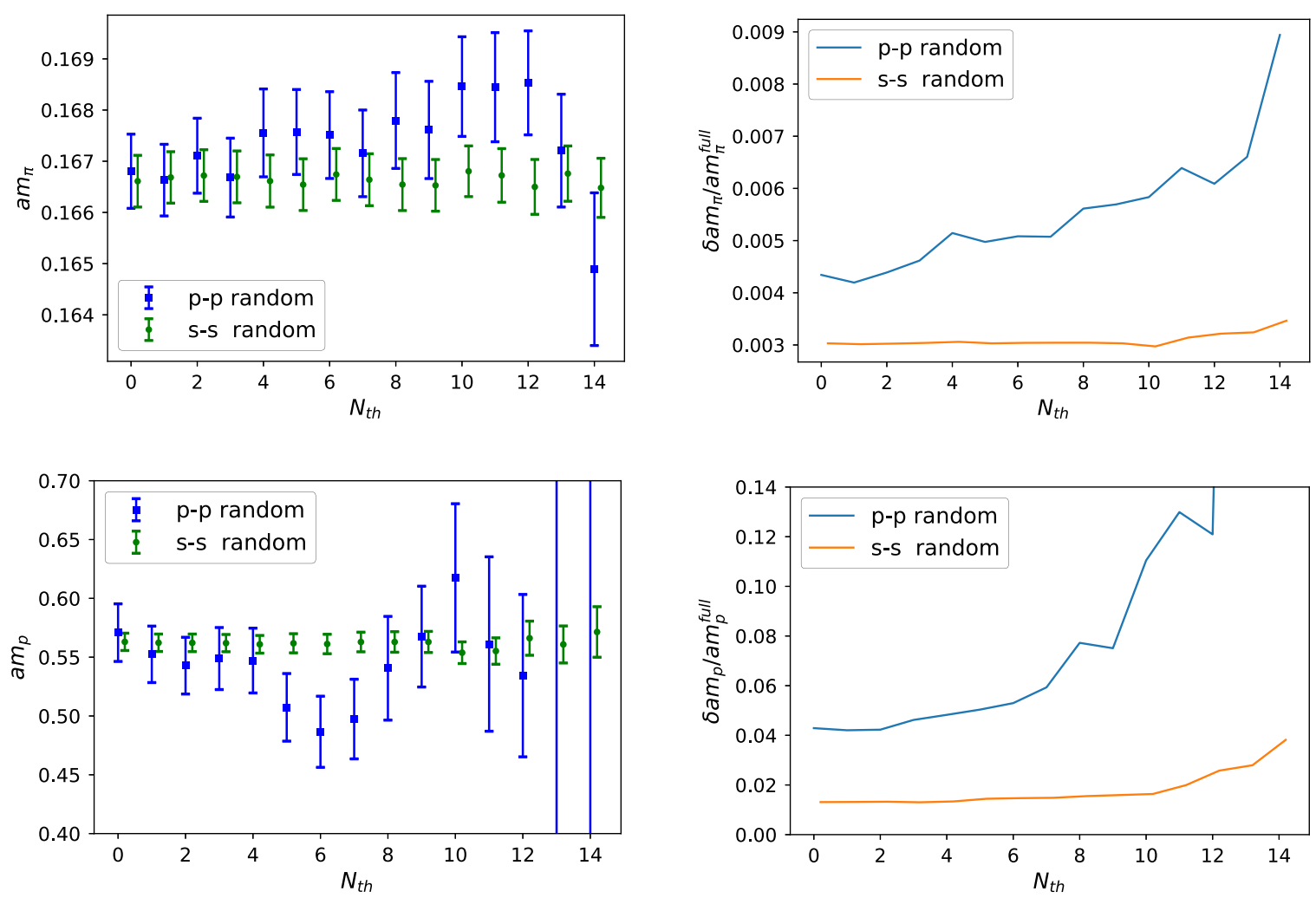

FIG. 3. Effective masses for the pion and proton from the smeared-source smeared-sink (s-s) and point-source point-sink (p-p) correlation functions. In the left panel, the effective masses as a function of $N_{\mathrm{th}}$ are shown. Here we use the random field selection. In the right panel, the ratio between the statistical uncertainty and the effective mass $m_{\alpha}^{\text {full }}$ for $\alpha=\pi$ and $p$ is shown.

to 1 for both smeared-source smeared-sink (s-s) and pointsource point-sink (p-p) correlation functions. The effective masses from the correlated fit are shown in the left panels of Fig. 3, while the ratios between the statistical uncertainty and the effective masses $m_{\alpha}^{\text {full }}$ for $\alpha=\pi$ and $p$ are shown in the corresponding right panels. With the same statistics, the results of $p-p$ correlators are noisier than that of the s-s ones. The field-sparsening method works less efficiently in the p-p correlators as we expect. Nevertheless, we find that at $N_{\text {th }}=5\left(N_{\Lambda}=108\right)$ the uncertainty of the p-p effective mass increases only $15 \%$ for the pion and $18 \%$ for the proton. This implies that one can reduce the field points by a factor of 128 in trade with a small increase of the statistical error. Two orders of magnitude reduction in the propagator storage and I/O data transfer would make a typical lattice calculation much easier.

\section{NOISE FROM RANDOM FIELD SELECTION METHOD}

When using the random field selection method, the observable $\mathcal{O}$ depends on the gauge field $U$ and the random field selection $\Lambda$. The expectation value of the observable is

$$
C=\mathrm{E}_{\Lambda}\left[\int \mathcal{D} U \frac{e^{-S[U]}}{Z} \mathcal{O}(U, \Lambda)\right],
$$

where $Z=\int \mathcal{D} U e^{-S[U]}, \Lambda$ stands for the random field selection, and the operator $\mathrm{E}_{\Lambda}$ evaluates the expectation value of the expression in $[\cdots]$ as a function of the random variable $\Lambda$. In the random field selection method, we use independent random selection for each gauge field configuration

$$
C \approx \frac{1}{N_{\text {conf }}} \sum_{i=1}^{N_{\text {conf }}} \mathcal{O}\left(U_{i}, \Lambda_{i}\right) .
$$

The statistical uncertainty $\delta$ is given by

$$
\begin{gathered}
\delta^{2}=\frac{1}{N_{\text {conf }}} \mathrm{E}_{\Lambda}\left[\int \mathcal{D} U \frac{e^{-S[U]}}{Z}(\mathcal{O}(U, \Lambda)-C)^{2}\right] \\
\approx \frac{1}{N_{\text {conf }}} \sum_{i=1}^{N_{\text {conf }}}\left(\mathcal{O}\left(U_{i}, \Lambda_{i}\right)-C\right)^{2} .
\end{gathered}
$$

It can de decomposed into two parts

$$
\delta^{2}=\delta_{\text {gauge }}^{2}+\delta_{\text {rand }}^{2},
$$

where 


$$
\begin{gathered}
\delta_{\text {gauge }}^{2}=\frac{1}{N_{\text {conf }}} \mathrm{E}_{\Lambda}\left[\int \mathcal{D} U \frac{e^{-S[U]}}{Z}\left(\mathcal{O}(U, \Lambda)-\int \mathcal{D} U^{\prime} \frac{e^{-S\left[U^{\prime}\right]}}{Z} \mathcal{O}\left(U^{\prime}, \Lambda\right)\right)^{2}\right], \\
\delta_{\text {rand }}^{2}=\frac{1}{N_{\text {conf }}} \mathrm{E}_{\Lambda}\left[\left(\int \mathcal{D} U \frac{e^{-S[U]}}{Z} \mathcal{O}(U, \Lambda)-C\right)^{2}\right] .
\end{gathered}
$$

The first part is the gauge noise, $\delta_{\text {gauge }}$, which arises from the Monte-Carlo method used to perform the path integral over the gauge field. The second is the noise from the random selection of the field points, $\delta_{\text {rand }}$. Both $\delta_{\text {gauge }}$ and $\delta_{\text {rand }}$ vanish in the infinite configurations limit. Note that the gauge noise $\delta_{\text {gauge }}$ accounts for the increase of noise due to the reduction of statistics by selecting less field points than the full set, while $\delta_{\text {rand }}$ is responsible for additional noise from the random selection. The increase of the gauge noise is an inevitable price when the number of the field points is reduced in the summation. Since the lattice data at the different points are highly correlated, we expect the gauge noise only increases mildly. We therefore focus on the estimation of $\delta_{\text {rand }}$.

We write the time dependence of the 2-point correlation function as

$$
\begin{aligned}
C(\vec{x}, t) & =\left\langle O(\vec{x}, t) O^{\dagger}(\overrightarrow{0}, 0)\right\rangle \\
& \doteq \frac{1}{L^{3}} \sum_{\vec{p}} e^{i \vec{p} \cdot \vec{x}} \frac{\tilde{A}(\vec{p})}{2 E}\left(e^{-E t}+e^{-E(T-t)}\right),
\end{aligned}
$$

where the symbol of $\doteq$ reminds us that the excited-state contributions are neglected at sufficiently large $t$. The energy $E$ satisfies the dispersion relation $E=\sqrt{m^{2}+\vec{p}^{2}}$ with $m$ the hadron's mass.

To fully understand the impact on the uncertainty of the correlation function from the random field selection, one needs to determine the weight function $\tilde{A}(\vec{p})$. Considering the fact that we have used the Gaussian-smeared source and sink in our calculation, we assume that the weight function in the coordinate space is given by a Gaussian distribution

$$
A(\vec{x})=A_{0} e^{-\vec{x}^{2} / \sigma^{2}} .
$$

Then the weight function in the momentum space is given by the Fourier transformation of $A(\vec{x})$

$$
\tilde{A}(\vec{p})=\int d^{3} \vec{x} e^{-i \vec{p} \cdot \vec{x}} A(\vec{x})=A_{0}\left(\frac{\pi}{\sigma}\right)^{\frac{3}{2}} e^{-\frac{\sigma^{2}}{4} \vec{p}^{2}}
$$

In the following context we use the pion correlation function as an example. The analysis for the case of the proton is similar. Plugging Eq. (19) into Eq. (17) we can use the resulting formula to fit the pion lattice correlator with three free parameters, namely $A_{0}, \sigma$ and $m$. We obtain $m=0.165(1)$ which is consistent with the effective mass $m_{\pi}^{\text {full }}$ calculated before. Furthermore, we can obtain the result for $C(r, t)$ by averaging the lattice data of $C(\vec{x}, t)$ in a range of $r \leq|\vec{x}|<r+1$. In Fig. 4 we show the $C(r, t)$ at $t / a=10$ together with the fitting curve. The excited-state contribution can be safely neglected at $t / a=10$ and thus the fit interval can be chosen as $0 \leq r / a \leq 20$. In practice we introduce a truncation $p_{\max }=3(2 \pi / L)$ for the momentum summation in Eq. (17). But this is not very necessary as contributions from large momenta are suppressed significantly by the factor of $e^{-E t}+e^{-E(T-t)}$ at $t / a=10$. The good agreement suggests that the functional form given in Eq. (17) describes the lattice data well at large spacetime distances.

As a next step, we use the parameters $A_{0}, \sigma$ and $m$ and Eqs. (17) and (19) to construct the correlation function $\hat{C}(\vec{x}, t)$. As the gauge noise is eliminated in $\hat{C}(\vec{x}, t)$, we can isolate the noise $\delta_{\text {rand }}$ by replacing the lattice correlator $C(\vec{x}, t)$ with $\hat{C}(\vec{x}, t)$ in the random field selection method. In Fig. 5 we compare the uncertainties for the three different types of 2-point functions at $t=10,15$ and 20: The blue bar indicates the uncertainty of the correlation function with random field selection method with $N_{\Lambda}=1$. The orange bar shows the uncertainty for the data with $N_{\Lambda}=\Lambda_{\text {full }}$ while the green bar indicates the size of $\delta_{\text {rand }}$ only. It is noticed that the size of $\delta_{\text {rand }}$ is much smaller than the gauge noise and thus can be safely neglected. We have thus reached a conclusion that the precision of the pion 2-point functions using the random field selection method is equally good as that using the sparse-grid method. The random field selection is

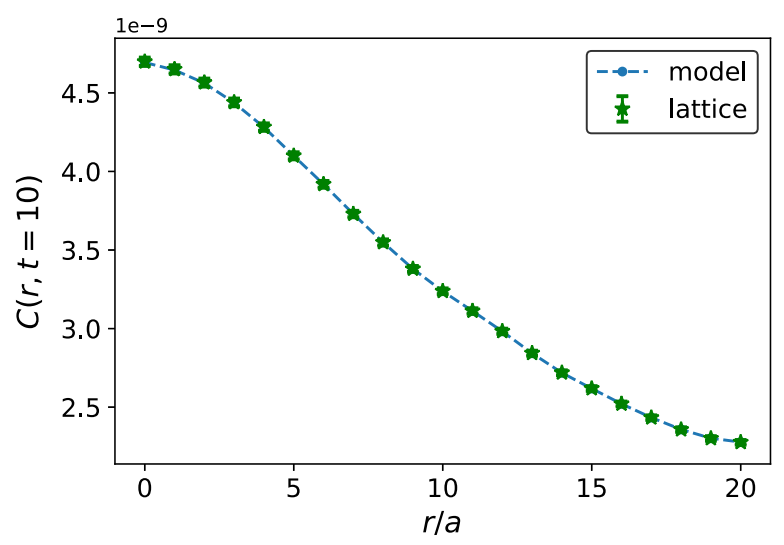

FIG. 4. Lattice results of $C(r, t)$ at $t=10$ together with a fit to Eq. (17). 

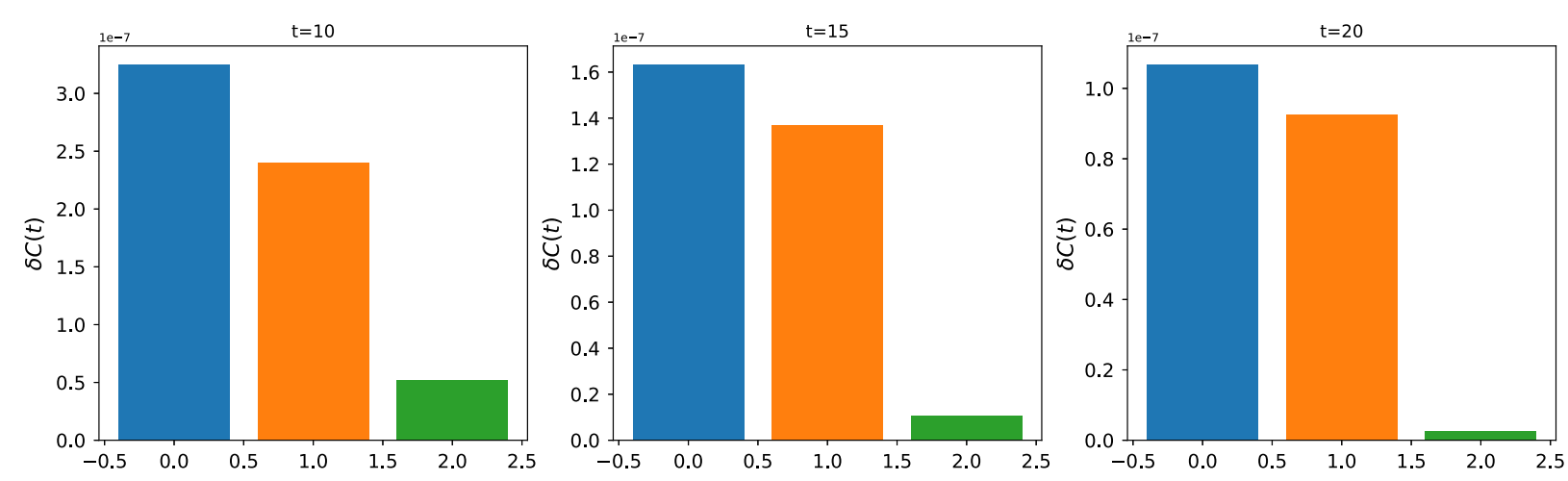

FIG. 5. Uncertainties for the three types of 2-point functions at $t=10,15$ and 20 . The blue bar indicates the uncertainty of the correlation function with random field selection for $N_{\Lambda}=1$. The orange bar shows the uncertainty for the data using $\Lambda_{\text {full }}$. The green bar shows the $\delta_{\text {rand }}$ only.

theoretically cleaner as there is no enhancement of the excited-state contamination.

\section{3-POINT FUNCTION}

In this section, we extend the random field selection method to the 3-point function, where we calculate the proton axial charge $g_{A}$ as an example which is one of the most fundamental quantities in nuclear physics. A global average of the lattice results of $g_{A}$ can be found in the latest FLAG review [12]. In this study we mainly focus on the efficiency of the random field selection method rather than the full control of various systematic effects for $g_{A}$. We start with the 3-point and 2-point functions as

$$
C^{3 \mathrm{pt}}\left(t_{i}, t_{s}\right)=\frac{L^{6}}{N_{\Lambda} N_{\Lambda_{s}} N_{\Lambda_{0}} N_{\Lambda_{t}}} \sum_{\vec{x} \in \Lambda} \sum_{\vec{x}_{s} \in \Lambda_{s}} \sum_{\vec{x}_{0} \in \Lambda_{0}} \sum_{t_{0} \in \Lambda_{t}}\left\langle\mathcal{P}\left[O_{p}\left(t_{0}+t_{s}, \vec{x}_{s}\right) A_{3}\left(t_{0}+t_{i}, \vec{x}\right) O_{p}^{\dagger}\left(t_{0}, \vec{x}_{0}\right)\right]\right\rangle
$$

and

$$
C^{2 \mathrm{pt}}\left(t_{s}\right)=\frac{L^{3}}{N_{\Lambda_{s}} N_{\Lambda_{0}} N_{\Lambda_{t}}} \sum_{\vec{x}_{s} \in \Lambda_{s}} \sum_{\vec{x}_{0} \in \Lambda_{0}} \sum_{t_{0} \in \Lambda_{t}}\left\langle\mathcal{P}\left[O_{p}\left(t_{s}+t_{0}, \vec{x}_{s}\right) O_{p}^{\dagger}\left(t_{0}, \vec{x}_{0}\right)\right]\right\rangle,
$$

where $O_{p}$ is the Gaussian-smeared operator for the proton, $A_{3}$ is the axial vector current with the polarization in the $z$ direction and $\mathcal{P}$ is the spin projection operator.

In order to compare the field-sparsening results with the full-size ones, we use the sequential-source propagators, which start from the source $\left(t_{0}, \vec{x}_{0}\right)$, go through the current insertion point $\left(t_{0}+t_{i}, \vec{x}\right)$ and end at the sink location $\left(t_{0}+t_{s}, \vec{x}_{s}\right)$. We use 24 time slices for $t_{0}$. For each $t_{0}$, one random source is used and the time $t_{i}$ and $t_{s}$ are fixed as $t_{i}=5$ and $t_{s}=10$. In total we generate 24 sequentialsource propagators for each configuration. These propagators allow us to obtain the 3-point functions with $N_{\Lambda_{0}}=1, N_{\Lambda_{t}}=24, N_{\Lambda}=L^{3}$ and arbitrary values of $N_{\Lambda_{s}}$. By comparing the lattice results at different $N_{\Lambda_{s}}$, one can estimate how large the correlation among the sink points in the 3-point function. In the current study, we fixed $t_{i}$ and $t_{s}$. The excited-state effects will be taken into consideration later. In Fig. 6, it shows the ratio between 3-point and 2-point functions, $C^{3 \mathrm{pt}} / C^{2 \mathrm{pt}}$, as a function of
$N_{\Lambda_{\mathrm{s}}}$ (the $x$-axis is labeled by $N_{\mathrm{th}}$ ). We find that at $N_{\mathrm{th}}=10$ $\left(N_{\Lambda_{s}}=16\right)$, the uncertainty of the ratio $C^{3 \mathrm{pt}} / C^{2 \mathrm{pt}}$ only increases by $12 \%$ compared to the case of $N_{\text {th }}=0$ $\left(N_{\Lambda_{s}}=L^{3}\right)$. This is very consistent with the observation in the effective masses of the 2-point functions. Thus we can conclude that the field-sparsening method seems to work equally well for both 2-point and 3-point functions.

As a next step, we want to determine the optimal values of $N_{\Lambda_{0}}$ and $N_{\Lambda_{s}}$ for the source and sink location points. Here the 3-point functions are constructed using the Gaussian-smeared propagators only, which start from both source and sink locations and end at the current insertion point $\left(t_{0}+t_{i}, \vec{x}\right)$. These Gaussian-smeared propagators are placed at 24 time slices, which results in $N_{\Lambda_{t}}=24$. At each time slice we calculate 32 Gaussian-smeared propagators. It allows us to build the correlators with the $N_{\Lambda_{0}}-N_{\Lambda_{s}}$ pair changing from 1-1 to 32-32. We perform the summation of current insertion location $\vec{x}$ over the whole spatial volume and have $N_{\Lambda}=L^{3}$. In the left panel of Fig. 7, we show the 

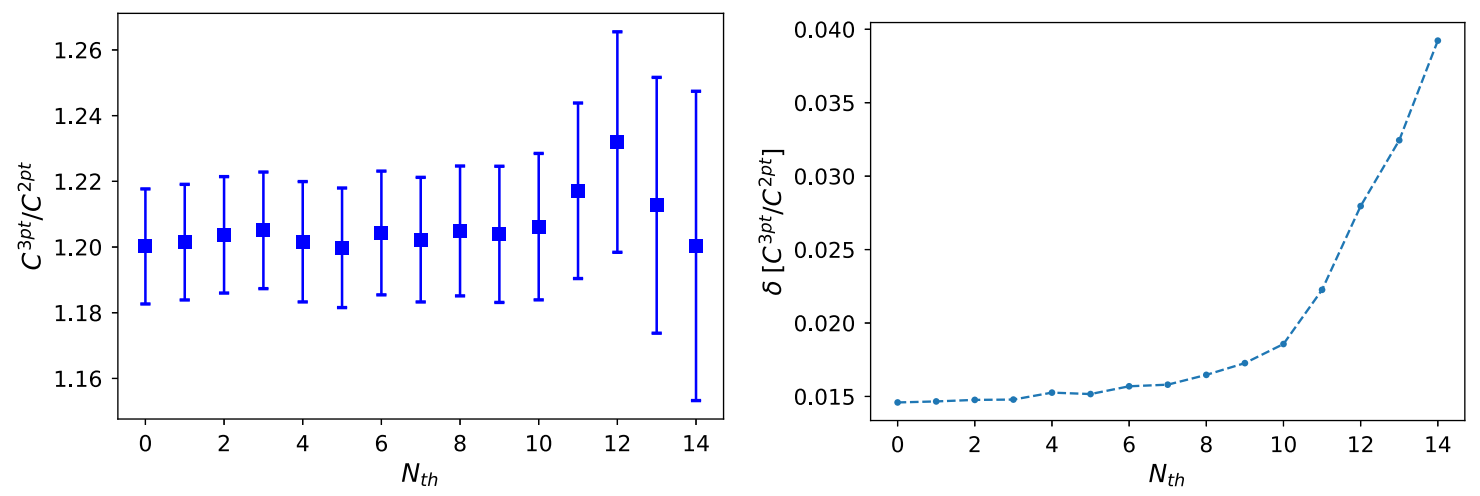

FIG. 6. The left panel shows the ratio between 3-point and 2-point functions, $C^{3 \mathrm{pt}} / C^{2 \mathrm{pt}}$, as a function of $N_{\Lambda_{\mathrm{s}}}$ (the $x$-axis is labeled by $\left.N_{\mathrm{th}}\right)$. The 3-point functions are constructed using the sequential-source propagators. The time separation $t_{i}$ and $t_{s}$ are fixed as $t_{i}=5$ and $t_{s}=10$. In the right panel, the uncertainty of the ratio as a function of $N_{\text {th }}$ is shown.
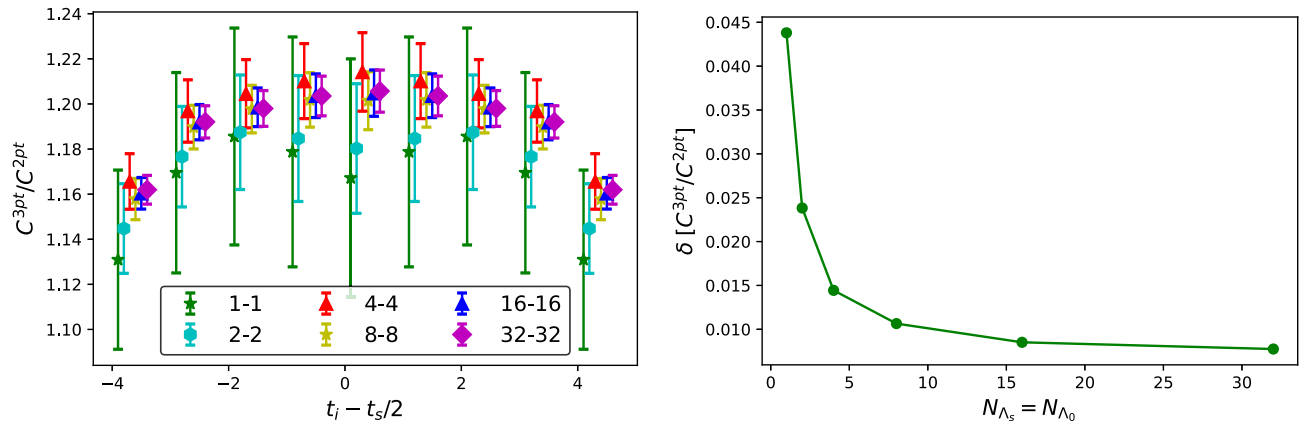

FIG. 7. The left panel shows the results of $C^{3 \mathrm{pt}} / C^{2 \mathrm{pt}}$ as a function of $t_{i}-t_{s} / 2$, where $t_{s}$ is chosen to be 10 . The right panel shows the uncertainty of the ratio (with $t_{s}=10$ and $t_{i}-t_{s} / 2=0$ ) as a function of $N_{\Lambda_{0}}=N_{\Lambda_{s}}$. In these two plots, we set $N_{\Lambda}=L^{3}$.

results of $C^{3 \mathrm{pt}} / C^{2 \mathrm{pt}}$ as a function of $t_{i}-t_{s} / 2$, where $t_{s}$ is chosen to be 10 and the values of $t_{i}-t_{s} / 2$ vary in the range of $[-4,4]$. The data points with various $N_{\Lambda_{0}}-N_{\Lambda_{s}}$ pairs are plotted using the different symbols. In the right panel of Fig. 7, the statistical uncertainty of $C^{3 \mathrm{pt}} / C^{2 \mathrm{pt}}$ as a function of $N_{\Lambda_{0}}=N_{\Lambda_{s}}$ are shown. We find that with $N_{\Lambda_{0}}$ changing from 1 to 4 , the statistical error drops relatively fast. From $N_{\Lambda_{0}}=4$ to 8 , the error decreases slower. Due to the high correlation, the change of the uncertainty is very mild from $N_{\Lambda_{0}}=8$ to 32. It is unnecessary to move on to larger $N_{\Lambda_{0}}$ as one can expect that the precision at $N_{\Lambda_{0}}=N_{\Lambda_{s}}=8$ is close to the best precision using the all-to-all setup $\left(N_{\Lambda_{0}}=\right.$ $\left.N_{\Lambda_{s}}=L^{3}\right)$. In practice, we can choose $N_{\Lambda_{0}}=4$ or 8 and invest the additional computational resources in accumulating data from more gauge configurations.

In Fig. 7, the ratio of $C^{3 \mathrm{pt}} / C^{2 \mathrm{pt}}$ at $t_{s}=10$ is shown. As a next step we add the lattice results at another two values of $t_{s}\left(t_{s}=8\right.$ and 12) and vary $t_{i}-t_{s} / 2$ in a range of $\left[-t_{s}+1, t_{s}-1\right]$. The corresponding results are shown in Fig. 8. At large time separation, the time dependence of $C^{3 \mathrm{pt}} / C^{2 \mathrm{pt}}$ can be approximated by a two-state form as

$\frac{C^{3 \mathrm{pt}}\left(t_{i}, t_{s}\right)}{C^{2 \mathrm{pt}}\left(t_{s}\right)}=\frac{g_{A}+c_{1} e^{-\Delta t_{s}}+c_{2}\left(e^{-\Delta\left(t_{s}-t_{i}\right)}+e^{-\Delta t_{i}}\right)}{1+c_{0} e^{-\Delta t_{s}}}$, where $\Delta$ is the energy difference between the excited state and the ground state. The coefficients of $c_{0}, c_{1}$ and $c_{2}$ arise from the excited-state contamination. We determine the values of $\Delta$ and $c_{0}$ from the 2-point function and then perform a two-state fit of $C^{3 \mathrm{pt}} / C^{2 \mathrm{pt}}$ to Eq. (22) using three free parameters $g_{A}, c_{1}$ and $c_{2}$. The lattice data for three $t_{s}$

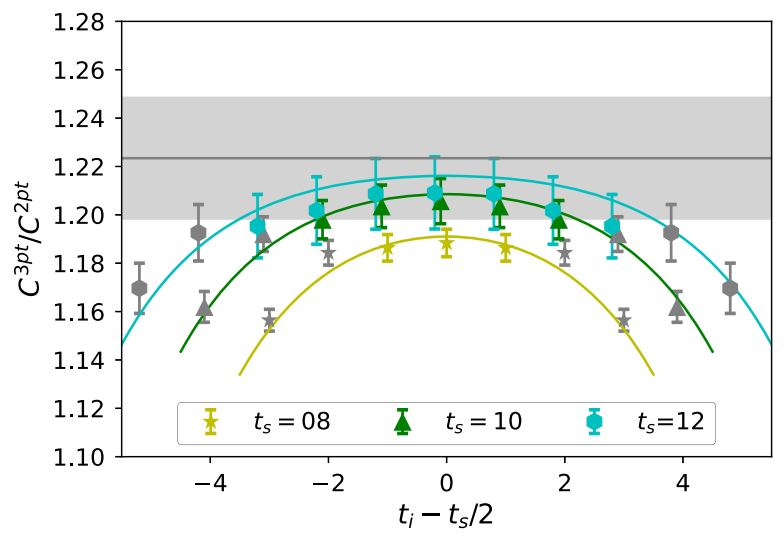

FIG. 8. The ratio of $C^{3 \mathrm{pt}} / C^{2 \mathrm{pt}}$ as a function of $t_{i}-t_{s} / 2$ together with a two-state fit. The shadowed band indicates the result of $g_{A}$ obtained from the fit. We use $N_{\Lambda_{s}}=N_{\Lambda_{0}}=32, N_{\Lambda}=L^{3}$, and $N_{\Lambda_{t}}=24$. 


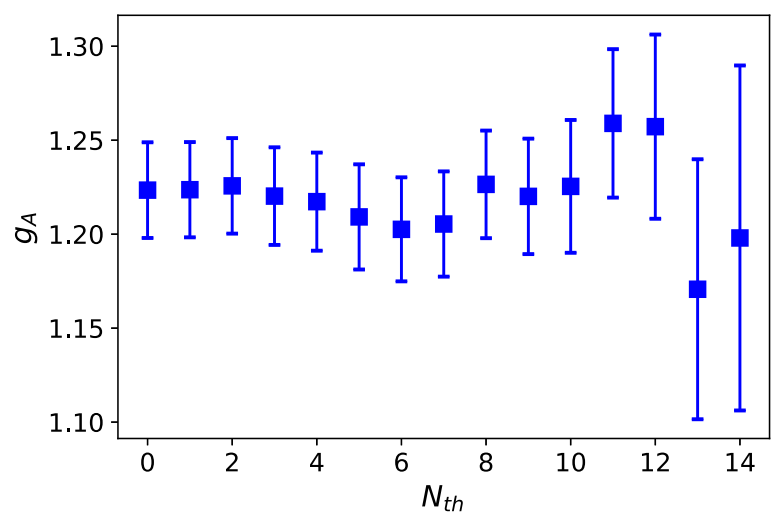

FIG. 9. The proton axial charge $g_{A}$ as a function of $N_{\text {th }}$. Here $N_{\text {th }}$ is related to the number of field points at the location of current insertion. We use $N_{\Lambda_{s}}=N_{\Lambda_{0}}=32$ and $N_{\Lambda_{t}}=24$.

are used in the fit simultaneously with $t_{i}-t_{s} / 2$ ranging from $\left[-t_{s} / 2+3, t_{s} / 2-3\right]$. As a final result we obtain the axial charge $g_{A}=1.223(25)$ at the pion mass $m_{\pi} \approx$ $350 \mathrm{MeV}$ using 91 configurations and $N_{\Lambda_{s}}=N_{\Lambda_{0}}=32$, $N_{\Lambda}=L^{3}$, and $N_{\Lambda_{t}}=24$ for each configuration.

As a last step, we calculate $g_{A}$ with different value of $N_{\Lambda}$, which is the number of the field points at the location of current insertion $\left(t_{0}+t_{i}, \vec{x}_{i}\right)$. In Fig. 9 we show $g_{A}$ as a function of $N_{\text {th }}$. By increasing $N_{\text {th }}$ from 0 to 5 (or equivalently reducing $N_{\Lambda}$ from $L^{3}$ to 108), we find that the uncertainty of $g_{A}$ only increases by $10 \%$. Therefore, by using the field sparsening, one can reduce the size of the Gaussian-smeared source point-sink propagators by a factor of 128 at the expense of $10 \%$ increase in the statistical error of the correlation function.

\section{CONCLUSION}

In this work we make an exploratory study on the fieldsparsening methods. The observables under investigation include the pion and proton 2-point correlation functions and the proton axial charge $g_{A}$ involving the 3-point functions. For the sparse-grid method, the results are not affected by the noise from field sparsening but receive additional excited-state contamination from the highermomenta states. For the random field selection method, the situation is just the opposite. There is no enhancement of the excited-state contamination, but the correlation functions are affected by the noise from random selection. Fortunately, we confirm, in both numerical lattice results and a model analysis mimicking pion correlator, that the noise from the random selection can be safely neglected.

In the calculation, we construct the correlation function using both Gaussian-smeared operator and the point-source operator. We find that Gaussian-smeared correlators do have higher correlation than the point-source ones. At the expense of a $\sim 15 \%$ increase in the statistical error, we can reduce the number of field points by a factor of $\sim 100$ for the point-source operator and a factor of $\sim 1000$ for the Gaussian-smeared operator. This is a surprisingly significant reduction in the size of the propagator. It also makes the storage of propagators much easier and saves the time for both I/O and quark contraction. Another interesting observation is that by using the field-sparsening methods, one can approach the precision of full-volume averaging with the modest cost of the computational resources. Since the nucleon fields at source and sink are symmetric, we thus demonstrate that field-sparsening method can approach the all-to-all limit rather quickly. Due to its high efficiency, we can foresee a vast application prospect of the fieldsparsening methods proposed in this paper.

\section{ACKNOWLEDGMENTS}

We thank ETM Collaboration for sharing the gauge configurations with us. X.F. and L.C.J. gratefully acknowledge many helpful discussions with our colleagues from the RBC-UKQCD Collaboration. X. F. and S. C.X. are supported in part by NSFC of China under Grant No. 11775002 . X.F. and C. L. are supported in part by NSFC of China under Grant No. 12070131001. L. C. J. acknowledges support by DOE Grant No. DE-SC0010339. Y.L. and C.L. are supported in part by CAS Interdisciplinary Innovation Team, NSFC of China under Grant No. 11935017, and the DFG and the NSFC through funds provided to the Sino-Germen CRC 110 "Symmetries and the Emergence of Structure in QCD", DFG Grant No. TRR 110 and NSFC Grant No. 11621131001. The calculation was carried out on TianHe-3 (prototype) at Chinese National Supercomputer Center in Tianjin.
[1] T. Blum, T. Izubuchi, and E. Shintani, Phys. Rev. D 88, 094503 (2013).

[2] T. Blum, N. Christ, M. Hayakawa, T. Izubuchi, L. Jin, and C. Lehner, Phys. Rev. D 93, 014503 (2016).

[3] S.-J. Dong and K.-F. Liu, Phys. Lett. B 328, 130 (1994).
[4] W. Wilcox, Noise methods for flavor singlet quantities, in Interdisciplinary Workshop on Numerical Challenges in Lattice QCD (Springer, Berlin, Germany, 2000), pp. 127141, https://inspirehep.net/literature/541015.

[5] G. S. Bali, H. Neff, T. Duessel, T. Lippert, and K. Schilling (SESAM Collaboration), Phys. Rev. D 71, 114513 (2005). 
[6] J. Foley, K. J. Juge, A. Ó Cais, M. Peardon, S. M. Ryan, and J.-I. Skullerud, Comput. Phys. Commun. 172, 145 (2005).

[7] W. Detmold et al., arXiv:1908.07050.

[8] A. Li et al. (xQCD Collaboration), Phys. Rev. D 82, 114501 (2010).

[9] G. Martinelli and C. T. Sachrajda, Nucl. Phys. B316, 355 (1989).
[10] S. Capitani et al., Proc. Sci. LAT2009 (2009) 095 [arXiv:0910.5578].

[11] C. Alexandrou et al., Phys. Rev. D 98, 054518 (2018).

[12] S. Aoki et al. (Flavour Lattice Averaging Group), Eur. Phys. J. C 80, 113 (2020). 